\title{
Sponge boundary condition for frequency-domain modeling
}

\author{
Changsoo Shin
}

\section{ABSTRACT}

Several techniques have been developed to get rid of edge reflections from artificial boundaries. One of them is to use paraxial approximations of the scalar and elastic wave equations. The other is to attenuate the seismic waves inside the artificial boundary by a gradual reduction of amplitudes. These techniques have been successfully applied to minimize unwanted seismic waves for time-domain seismic modeling. Unlike time-domain seismic modeling, suppression of edge reflections from artificial boundaries has not been successful in frequency-domain seismic modeling. Rayleigh waves caused by coupled motions of P- and $\mathrm{S}$-waves near the surface have been a particularly difficult problem to overcome in seismic modeling. In this paper, I design a damping matrix for frequencydomain modeling that damps out seismic waves by adding a diffusion term to the wave equation. This technique can suppress unwanted seismic waves, including Rayleigh waves and $\mathrm{P}$ - and $\mathrm{S}$-waves from an artificial boundary.

\section{INTRODUCTION}

Several numerical schemes that suppress artificial reflections from the boundaries of numerical models have been developed using the paraxial approximation of the wave equation (Reynolds, 1978; Clayton and Enquist, 1977). An alternative approach is to use a finite-thickness sponge to suppress edge reflections [Cerjan et al., 1985; Marfurt (1986, personal communication)]. Although the sponge technique has been applied to time-marching finite differences, finiteelement, and pseudospectral techniques, a boundary sponge for frequency-domain modeling has not been fully developed (Marfurt, 1984). The application of the Clayton and Enquist (1977) boundary condition in frequency-domain modeling results in an unsymmetric, complex impedance matrix (Pratt and Worthington, 1990) that doubles the computing time for factorization of the complex impedance matrix. In this paper, I present a definition of a damping term that will act like the numerical sponge condition in the time domain that has been developed by Marfurt (1984) and Cerjan et al. (1985).

\section{DESIGN OF DAMPING MATRIX FOR FREQUENCY DOMAIN MODELING}

Before discussing the sponge boundary condition in the frequency domain, the sponge boundary condition in the time domain will be reviewed. The sponge zone shown in Figure 1 will be used to damp out wave propagation. In this zone, the damping coefficient is 1.0 on the inside boundary $\Gamma_{i}$ and varies linearly to 0.90 on the outside boundary $\Gamma_{o}$. For example, the time-domain, finite-element formulation for scalar and elastic wave equation can be given (Marfurt, 1984) as

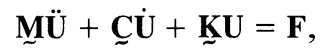

where $\mathbf{M}$ is a mass matrix, $\underset{\mathbf{C}}{\mathbf{C}}$ is a damping matrix, $\underset{\sim}{\mathbf{K}}$ is a stiffness matrix, $\mathrm{U}$ is a displacement, $\mathrm{F}$ is a source vector, and a dot indicates a time derivative.

The mass matrix can be formed either in a lumped (point collocation, finite-difference like) or consistent (Galerkin) form, or in some linear combination of the two (Marfurt, 1984). Equation (1) can be solved for the unknown displacements in either the time domain or the frequency domain. The details for time marching solutions can be found in many text books (Zienkiewiez, 1977; Bathe and Wilson, 1976). Taking the Fourier transform of equation (1) gives

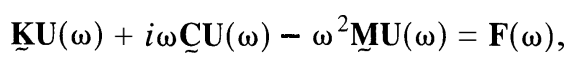

where $\omega$ is angular frequency, and $i=\sqrt{-1}$. Equation (2) can often can be expressed as 


$$
\underline{\mathbf{S}} \mathbf{U}(\omega)=\mathbf{F}(\omega)
$$

where the complex impedance matrix $\underline{\mathbf{S}}$ is given by

$$
\underline{\mathbf{S}}=\underline{\mathbf{K}}+i \omega \underline{\mathbf{C}}-\omega^{2} \underline{\mathbf{M}}
$$

Approximating $\ddot{\mathbf{U}}$ and $\dot{\mathbf{U}}$ by a central difference equation and introducing these difference equations into equation (1) yields

$$
\begin{aligned}
\left(\frac{\underline{\mathbf{M}}}{\Delta t^{2}}+\frac{\underline{\mathbf{C}}}{2 \Delta t}\right) \mathbf{U}(t+\mathrm{At}) & =\mathrm{F}-\underline{\mathbf{K}} \mathbf{U}(t)+2 \frac{\underline{\mathrm{M}}}{\Delta t^{2}} \mathbf{U}(t) \\
& -\frac{\underline{\mathrm{M}}}{\Delta t^{2}} \mathrm{U}(\mathrm{t}-\mathrm{At})+\frac{\underline{\mathrm{C}}}{2 \Delta t} \mathrm{U}(t-\mathrm{At}) .
\end{aligned}
$$

Multiplying equation (4) by $\Delta t^{2} / \mathbf{M}$ gives

$$
\begin{aligned}
& \left(1+\frac{\underline{\mathbf{C}}}{2 \underline{\mathbf{M}}} \Delta t\right) \mathbf{U}(t+\Delta t)=\mathbf{F}^{*}+2 \mathbf{U}(t)-\mathbf{U}(t-\Delta t) \\
& +\frac{C \Delta t}{2 \mathbf{M}} \mathbf{U}(t-\Delta t)
\end{aligned}
$$

where

$$
\mathbf{F}^{*}=\mathrm{F}+\frac{\underline{K} \mathbf{U}(t)}{\underline{\mathbf{M}}} \Delta t^{2} .
$$

Dividing equation (5) by $(1+\mathbf{C} / 2 \mathbf{M} A t)$ gives

$$
U\left(t+\mathrm{At}>=\frac{1}{1+\frac{\underline{\mathrm{C}}}{2 \underset{\sim}{\mathbf{M}}} \mathrm{At}} \mathrm{F}^{*}+\left(\frac{2}{1+\frac{\underline{\mathrm{C}}}{2 \underline{\mathbf{M}}} \Delta t}\right) \mathbf{U}(t)\right.
$$

$$
-\left(\frac{1}{1+\frac{\mathbf{C}}{2 \underline{M}} \Delta t}\right) \mathbf{U}(t-\mathrm{At})+\frac{1}{1+\frac{\mathscr{C}}{2 \underline{\mathbf{M}}} A t} \mid \cdot \frac{C \Delta t}{2 \mathbf{M}} \mathrm{U}(\mathrm{t}-A t)
$$

The damping coefficients (f) in the time domain are then

$$
f=\frac{1}{1+\frac{\mathbf{C}}{2 \mathbf{M}} \Delta t} .
$$

Suppose that the damping function from the artificial boundary to the inside zone is $f$, then the damping matrix $\mathbf{C}$ can be defined as follows

$$
\mathbf{C}=\frac{1-f}{f} \frac{2 \mathbf{M}}{\Delta t},
$$

where $f$ is a damping function that linearly varies from 0.98 to 1.0 in the time domain and from 0.9 to 1.0 in the frequency domain. It must be stated that the larger the sponge zone is, the smaller the damping coefficient will be. For example, the damping coefficient of the starting sponge zone in the time domain can be 0.98 , which requires more grid points in the $\mathrm{x}$ and z-direction, whereas the damping coefficient in the frequency domain can be 0.90 .

Since the mass matrix contains only a density term, the damping matrix $\mathrm{C}$ can be given for frequency domain finitedifference modeling as

$$
\mathbf{C}=\frac{1-f}{f} \frac{\rho(x, z)}{\text { At } 1}
$$

If we add the damping matrix defined by equation (8) or (9) to the complex impedance matrix, we can have a similar

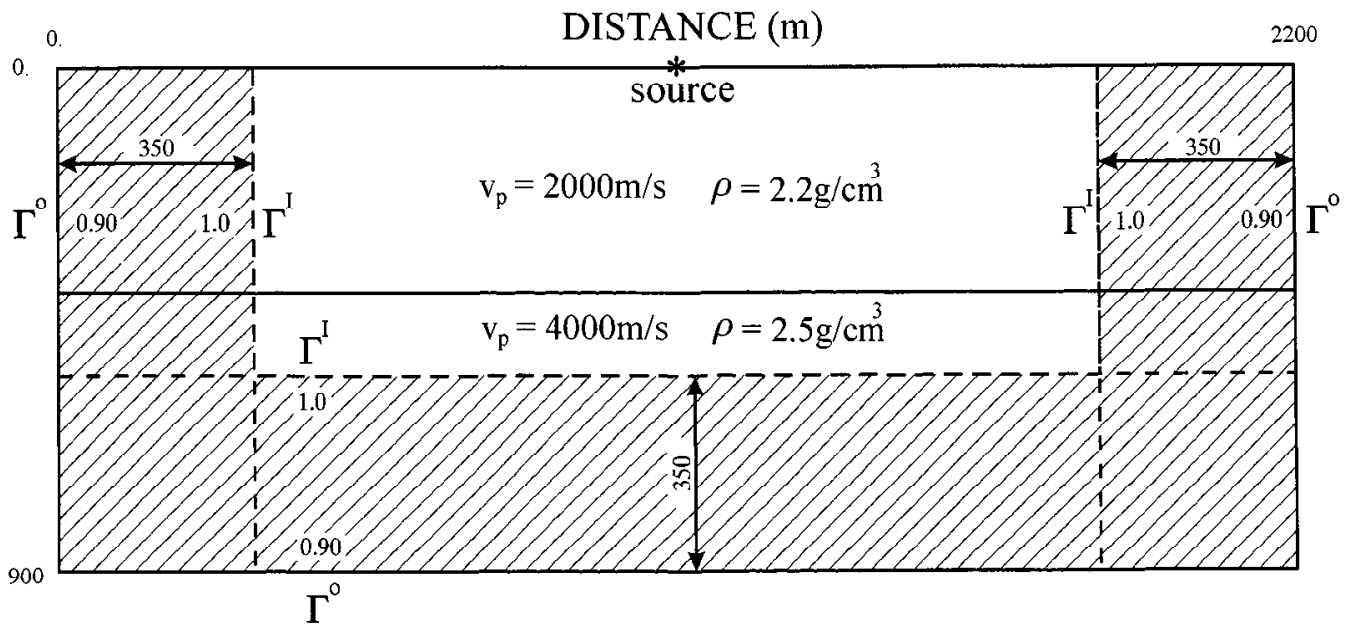

FIG. 1. Two layer model for testing boundary condition. The dashed line shows the sponge zone. The damping coefficient at the artificial boundary is 0.90 , and 30 grids are used to suppress the edge reflection. 
sponge effect to that produced in the time domain. It is important to choose At carefully, since it is the time sample interval used in performing the circular convolution that transforms frequency domain modeling data into time domain data.

\section{NUMERICAL EXAMPLE}

To test the validity of the sponge boundary condition in the frequency domain, the two-layer model shown in Figure 1 will be used. Figure 2 shows synthetic acoustic seismograms obtained with the Clayton and Enquist (1977) boundary condition; Figure 3 a shows acoustic seismograms using a sponge zone of 30 grids; and Figure $3 \mathrm{~b}$ shows acoustic seismograms using a sponge zone of 70 grids. Numerical tests indicate that 25 to 50 grid points are required to suppress the edge reflection in the acoustic and elastic frequency-domain modeling. This requirement increases the number of grid points by a factor of $1 \%$ to $20 \%$ in the $\mathrm{x}$ - and z-direction, depending upon the size of the given model. The second example considers elastic waves in the two-layer medium shown in Figure 4. The elastic seismograms calculated with the Clayton and Enquist (1977) boundary condition are shown in Figure 5. Figure 6 shows vertical and horizontal displacements generated by frequency-domain, finite-element modeling technique when using the sponge boundary condition. The source is a vertical force is applied on the free surface. Since the paraxial approximation of the elastic wave equation developed by Clayton and Enquist (1977) takes into account only P and S body waves, Rayleigh waves, which exist in the vicinity of a free surface cannot be suppressed efficiently by the paraxial approximation. Thus in Figure 5, the Rayleigh waves are not suppressed; however, the sponge boundary condition does efficiently attenuate the Rayleigh waves (Figure 6).

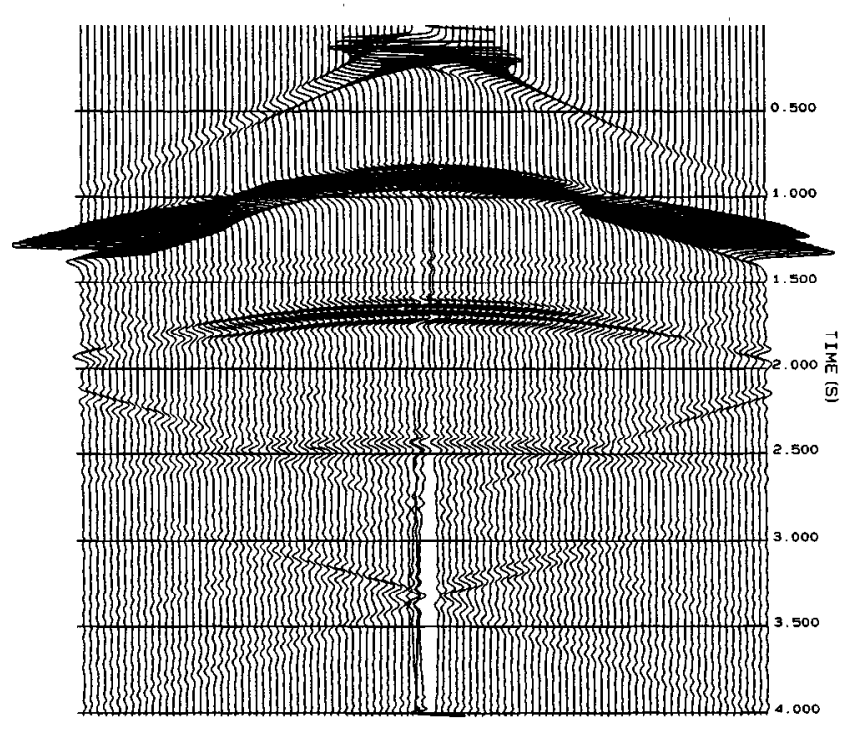

FIG. 2. Acoustic synthetic seismograms generated by frequency-domain, finite-element modeling technique using the Clayton and Enquist (1977) boundary condition.

\section{CONCLUSION}

I have presented a way of defining a damping matrix that mimics the effect of the sponge boundary condition for the suppression of edge reflections. The lumped mass matrix and the lumped damping matrix are employed in designing the damping matrix. As I increase the number of grids in the sponge zone, I obtain a better suppression of the artificial reflections. When dealing with strong Rayleigh artificial reflections, this approach can be an excellent choice for suppressing these surface waves. This damping matrix can be used in frequency-domain, finite-element and finite-difference modeling with only a small extra cost.

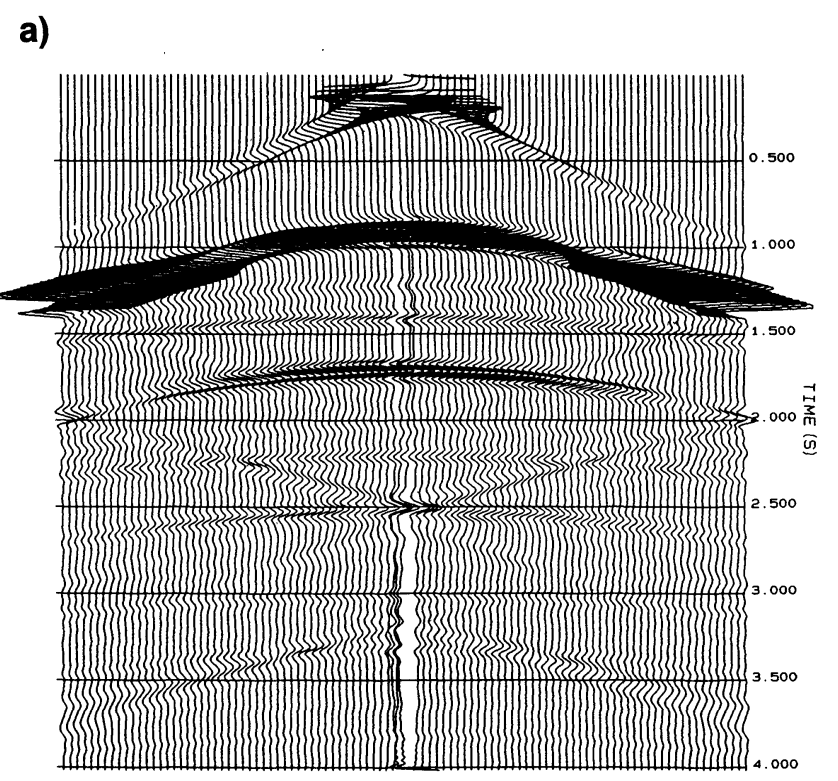

b)

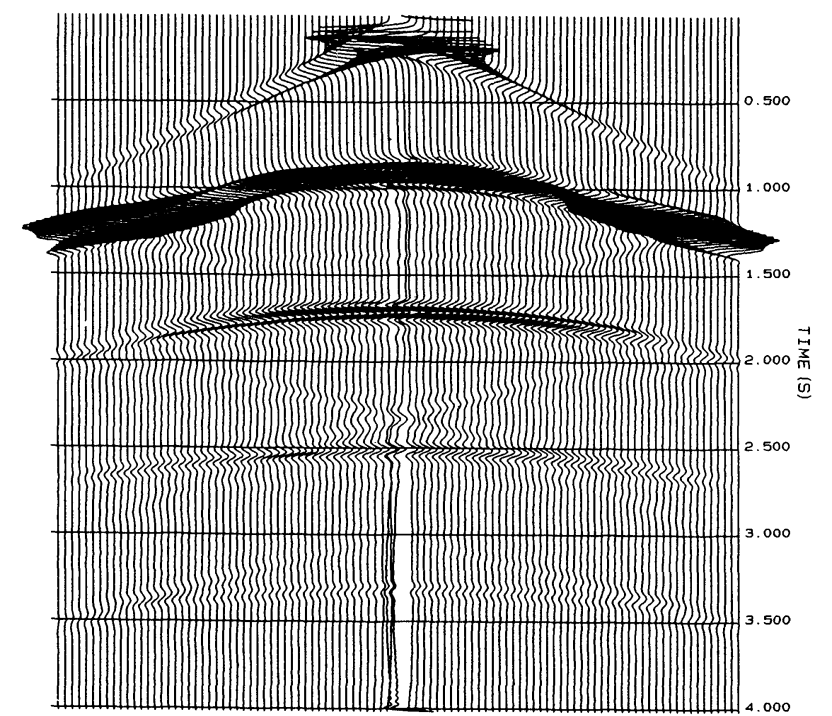

FIG. 3. (a) Acoustic synthetic seismograms generated by frequency-domain, finite-element modeling technique with a sponge zone of 30 grids. (b) Acoustic synthetic seismograms generated by frequency-domain, finite-element modeling technique with a sponge zone of 70 grids. 


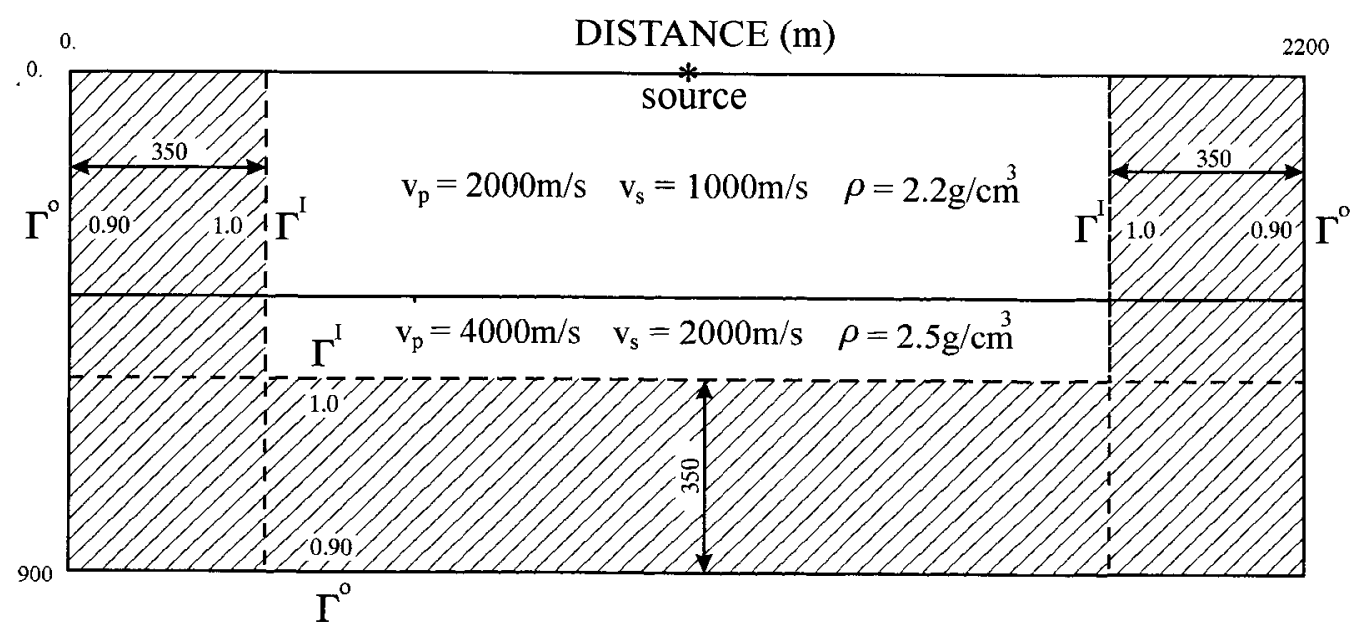

FIG. 4. Two-layer model used to test the boundary condition. The dashed line shows the sponge zone. The damping coefficient at the artificial boundary is 0.9 , and 35 grids are used to suppress the edge reflection.

a)

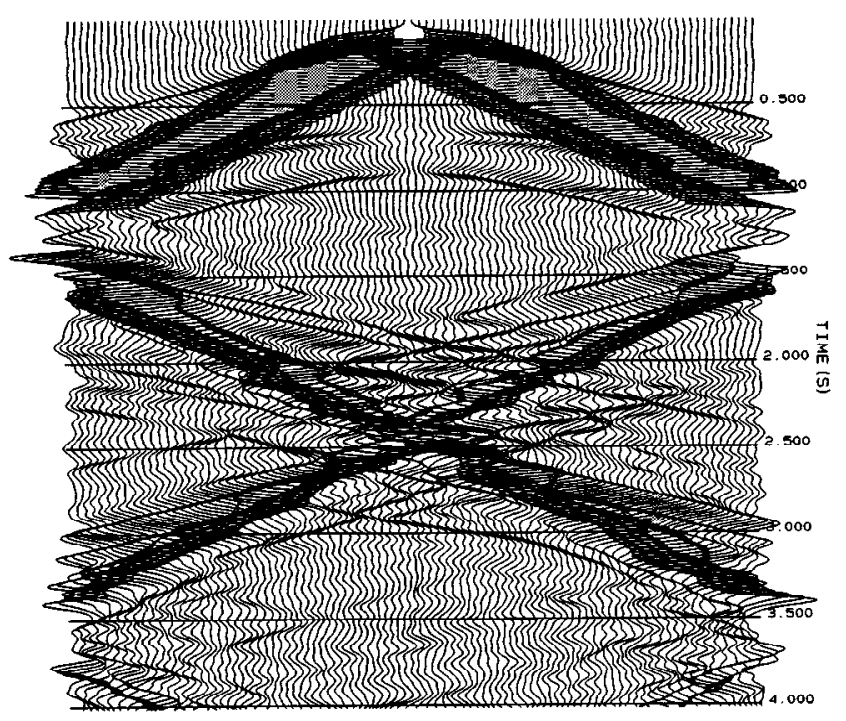

b)

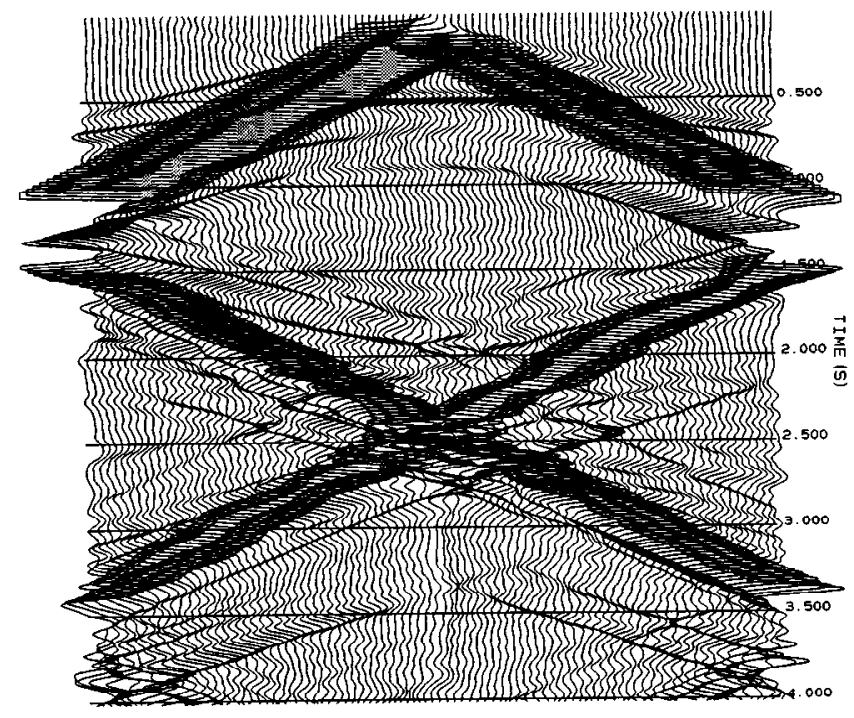

FIG. 5. (a) The horizontal displacement generated by frequency-domain, finite-element modeling technique using the Clayton and Enquist (1977) boundary condition. (b) The vertical displacement generated by frequency-domain, finite-element modeling technique using the Clayton and Enquist (1977) boundary condition. 
a)

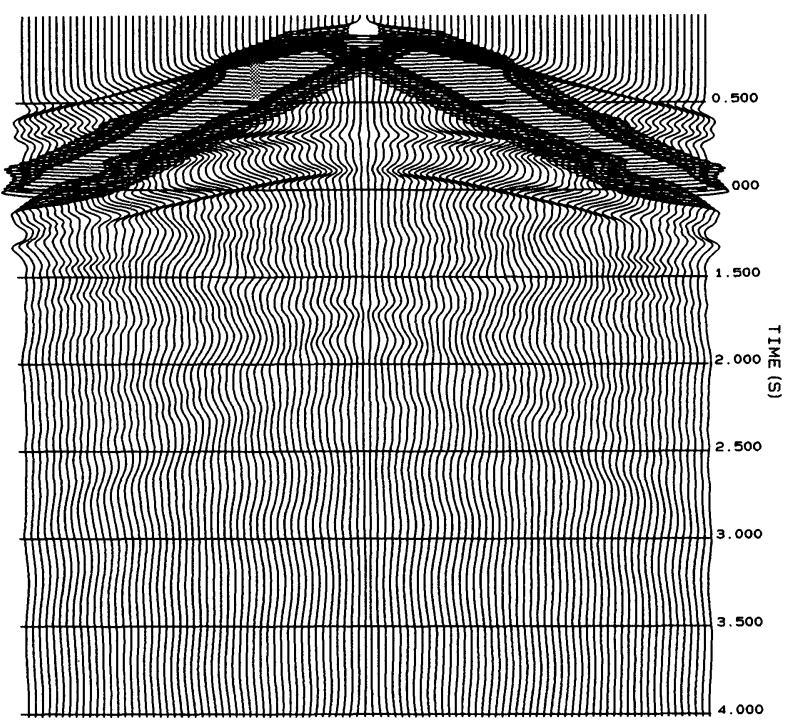

b)

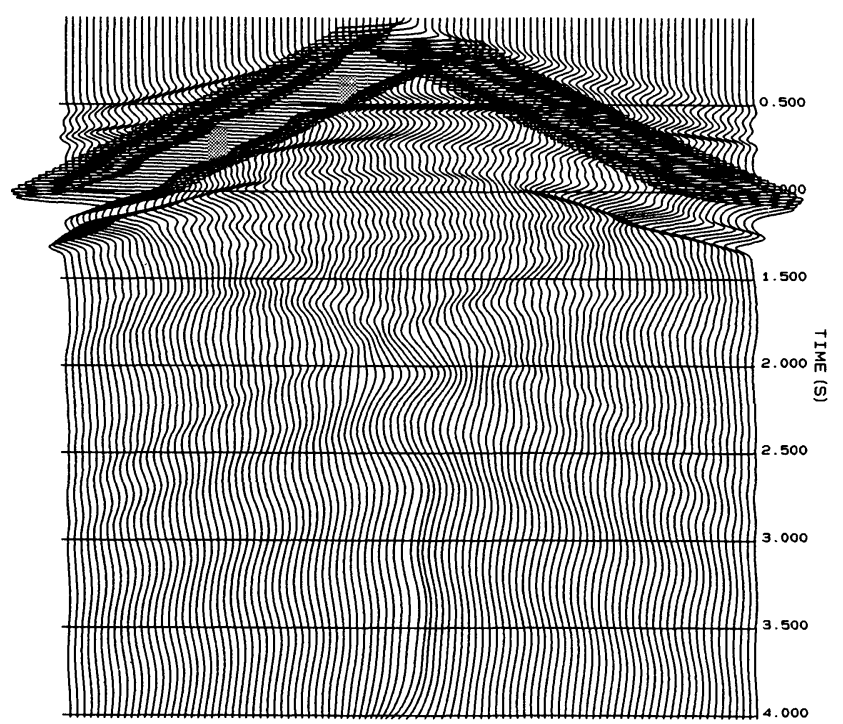

FIG. 6. (a) The horizontal displacement generated by frequency-domain, finite-element modeling using the sponge boundary condition. (b) The vertical displacement generated by frequency-domain, finite-element technique with the sponge boundary condition.

\section{REFERENCES}

Bathe, K. J., and Wilson, E. L., 1976, Numerical methods in finite-element analysis, Prentice Hall, Inc.

Cerjan, C., Kosloff, D., Kosloff, R., and Reshef, M., 1985, A nonreflecting boundary condition for discrete acoustic and elastic wave equation: Geophysics, 50, 705-708.

Clayton, R., and Enquist, B., 1977, Absorbing boundary conditions for acoustic and elastic wave equations: Bull. Seis. Soc. Am., 67, $1529-1540$.
Marfurt, K. J., 1984, Accuracy of finite-difference and finite-element modeling of the scalar and elastic wave equation: Geophysics, 49, 533-549.

Pratt, R. G., and Worthington, M. H., 1990, Inversion theory applied to multi-source crosshole tomography. Part 1: Acoustic wave equation, Geophys. Prosp., 38, 287-310.

Reynolds, A. C., 1978, Boundary conditions for the numerical solution of wave propagation problems: Geophysics, 43, 10991110.

Zienkiewiez, 0. C., 1977, The finite-element method, McGraw-Hill Book Co. 